\title{
Realization of a new large research infrastructure in Belgium: MYRRHA contribution for closing the nuclear fuel cycle making nuclear energy sustainable
}

\author{
Hamid AÏt Abderrahim $(*)$ \\ SCK CEN - Boeretang 200, B-2400 Mol, Belgium \\ UCLouvain, Ecole Polytechnique de Louvain \\ Place du Levant 2, B-1348 Louvain-la-Neuve, Belgium
}

\begin{abstract}
Summary. - In order to provide an appropriate level of energy to the whole world, nuclear energy is still going to play an important role. Nuclear energy can help reducing the $\mathrm{CO}_{2}$ emissions, which today are excessive. The problematics of nuclear waste can be solved using long-term geological storage in deep suitable formations. Partitioning and transmutation can help reducing the radiotoxicity of spent fuel to more acceptable durations of time. The MYRRHA project investigates since more than 20 years the possibility to demonstrate transmutation at a reasonable power level. In this paper we present the current state of the MYRRHA reactor design and the associated research and development activities.
\end{abstract}

\section{1. - Introduction}

1.1. Energy in the world. - One of the major challenges that our society faces is the increasing demand for energy in general and electricity in particular. According to international references (DOE/EIA, 2019, OECD, 2016) [1,2] global energy requirements

(*) E-mail: haitabde@sckcen.be 
will increase by $50 \%$ by 2035. Many factors play a role in this like the expected growth of world population, the availability of electricity to a part of the population that currently has not yet access to electricity, the higher consumption in countries that are still in full development today and the average life expectancy increase, to name a few.

During the last century, our energy supply was mainly based on fossil fuels. While the increase of the global energy requirement is in a continuing trend, the available sources of energy are also being increasingly tapped. Fossil fuels are still in sufficient supply, are easily accessible and at a reasonable price. However, we are confronted today with excessive $\mathrm{CO}_{2}$ emissions, so the use of fossil fuels needs to be limited. At the same time, renewable energy sources cannot fully satisfy the global demand. Therefore, different countries recognize that nuclear energy needs to be included in the "energy basket" of the future.

1'2. Nuclear energy. - Nowadays thermal nuclear reactors use nuclear fission of the fissile isotope of uranium (and in a smaller amount, plutonium) induced by thermal (slow) neutrons to generate heat and electricity. During this process, the fissile atom is split. The current nuclear workhorse for electricity production, the light water reactors, only fission a small portion of the raw uranium. When nothing changes from a technological point of view, the known uranium resources will become scarce around the end of the century (OECD/IAEA, 2018) [3] Reactors in which the neutrons have higher energies (so-called fast neutron reactors) increase the uranium utilization in the reactor and hence need a smaller amount of uranium ore per unit of energy generated. These reactors would allow using the remaining part of mineral uranium, leading to a more efficient use of the uranium resources.

During their operation present nuclear power reactors produce high-level radioactive waste (HLW). For this high-level radioactive waste, a technical and socially acceptable solution is necessary. Even though the solutions that are being considered at this moment, like geological disposal, might offer a technically valid solution, the time scale needed for the radiotoxicity to drop to the level of natural uranium remains very long, which is not acceptable for the public.

In nuclear fission, a uranium atom is split into two new, smaller nuclei, and, on average, two or three neutrons and about $200 \mathrm{MeV}$ of energy are released. The released neutrons can in turn split another uranium atom. This creates a chain reaction.

In a nuclear reactor the released neutrons from a fission reaction can undergo various reactions; they can induce a new fission leading to the birth of new neutrons, they can be absorbed in the structures of the reactor or even in the fuel without inducing fission leading to the production of heavier elements than uranium (like neptunium (Np), plutonium $(\mathrm{Pu})$, americium $(\mathrm{Am})$, curium $(\mathrm{Cm}), \ldots)$ or they can leak out of the reactor core and are considered lost to the chain reaction. Thus to maintain the chain reaction one speaks about a critical mass and critical dimensions of the reactor and these correspond to quantities of fissile materials arranged in a given geometry in such a way that the amount of neutrons produced by fissions is balanced by the amount of neutrons lost either by sterile absorptions or by leaking out of the reactor core. When these conditions 


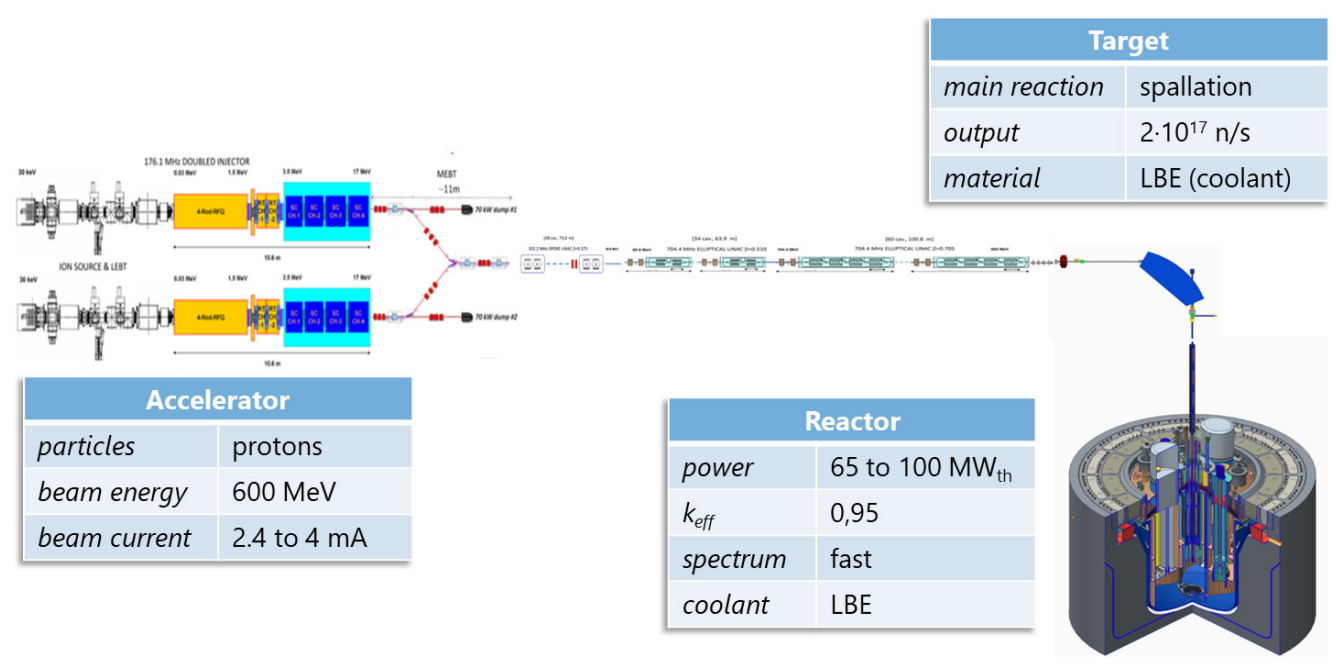

Fig. 1. - MYRRHA ADS coupling a Linac delivering $600 \mathrm{MeV} \times 4 \mathrm{~mA}$ proton beam in a $\mathrm{Pb}-\mathrm{Bi}$ cooled sub-critical reactor of $100 \mathrm{MWth}$.

are met, and the chain reaction is sustained by the system itself, one speaks about "critical" reactor.

An Accelerator-Driven System (ADS) will not have this self-sustained chain reaction. Indeed, its core is subcritical. In this context the term subcritical means that, on average, for each generation of neutrons, less than one secondary neutron is capable of initiating a subsequent nuclear fission. This situation can be achieved by limiting the amount of fissile material in the core, in this way the chain reaction is not self-sustaining. Despite this sub criticality situation one can operate such a reactor in continuous mode (i.e. constant power), but an external neutron source is needed. In an ADS, these supplementary neutrons are produced by spallation reactions, during which high-energy protons, coming from a particle accelerator (see fig. 1), are impinging on a spallation target made of a heavy metal like lead. In the course of the spallation process, the liquid metal nuclei emit a large number of neutrons whose energy spectrum is made of two parts: a conventional fission spectrum and a high-energy tail up to the energy of the incident proton. At $600 \mathrm{MeV}$, each impinging proton on a lead target would produce about 10 primary neutrons.

The generation of nuclear energy from uranium produces, besides energy, also highlevel nuclear waste. The HLW quantity one speaks about depends of the considered fuel cycle. To illustrate this let us consider the various fractions produced by using one ton of uranium as fuel in a Pressurized Water Reactor (PWR) thermal nuclear reactor after four and a half years (corresponding to an average burn up of $50000 \mathrm{MWd} / \mathrm{tHM}-1)$ : 
- $935 \mathrm{~kg}$ of uranium;

- $12 \mathrm{~kg}$ of plutonium;

- $2.4 \mathrm{~kg}$ of Minor Actinides (MAs), divided into

- $1 \mathrm{~kg}$ of neptunium;

$-0.8 \mathrm{~kg}$ of americium and

- $0.6 \mathrm{~kg}$ of curium;

- $\sim 50 \mathrm{~kg}$ of fission products (FP), from which a small part $(3.5 \mathrm{~kg}$ ) is long-lived (and called long-lived fission products or LLFPs).

\section{2. - Partitioning and transmutation}

Partitioning is the term used for the chemical processes needed to separate the different elements present in the spent nuclear fuel (actinides like uranium and plutonium; minor actinides like neptunium, americium and curium; fission products like cesium and strontium) such that a dedicated treatment can be applied to each stream. This is completely analogous to the concept of recycling in household waste: we separate paper from glass; compostable from plastic etc.

Transmutation is the process where we subject the (minor) actinides to a flux of fast, high energetic neutrons. Why choosing fast neutrons? Because at high energies, the probability that these neutrons induce a fission of these (minor) actinides, and hence transmute them into fission products, becomes higher than the probability that the neutron would be captured by the nucleus and the nucleus becomes an even heavier element (what would happen with slow, thermal neutrons).

Figure 2 illustrates the capture and fission cross-sections of ${ }^{241} \mathrm{Am}$ as a function of energy. We can see clearly that below $1 \mathrm{MeV}$ the capture cross-section is 2 to 3 orders of magnitude higher than the fission cross-section. The latter becomes predominant beyond a high-energy threshold a little bit below $1 \mathrm{MeV}(\sim 0.75 \mathrm{MeV})$.

Thus, it is obvious when considering MA's transmutation (fission and not capture) one needs to conduct it in fast neutron spectrum reactors. This is the first condition for a successful transmutation of MAs. Hence, transmutation requires systems that run on fast neutrons.

These fast neutron systems cannot be cooled by water since this would at the same time slow down the neutrons. Therefore, typical coolants for these fast neutron systems are liquid metals like sodium or lead/lead-bismuth or gases due to their low density such He.

Another issue that one has to consider when dealing with transmutation of minor actinides like neptunium, americium and curium in a critical fast neutron reactor is guaranteeing the reactivity safety control of the reactor. The reactivity control being driven by the weighted average value of the effective delayed neutron fraction of the 


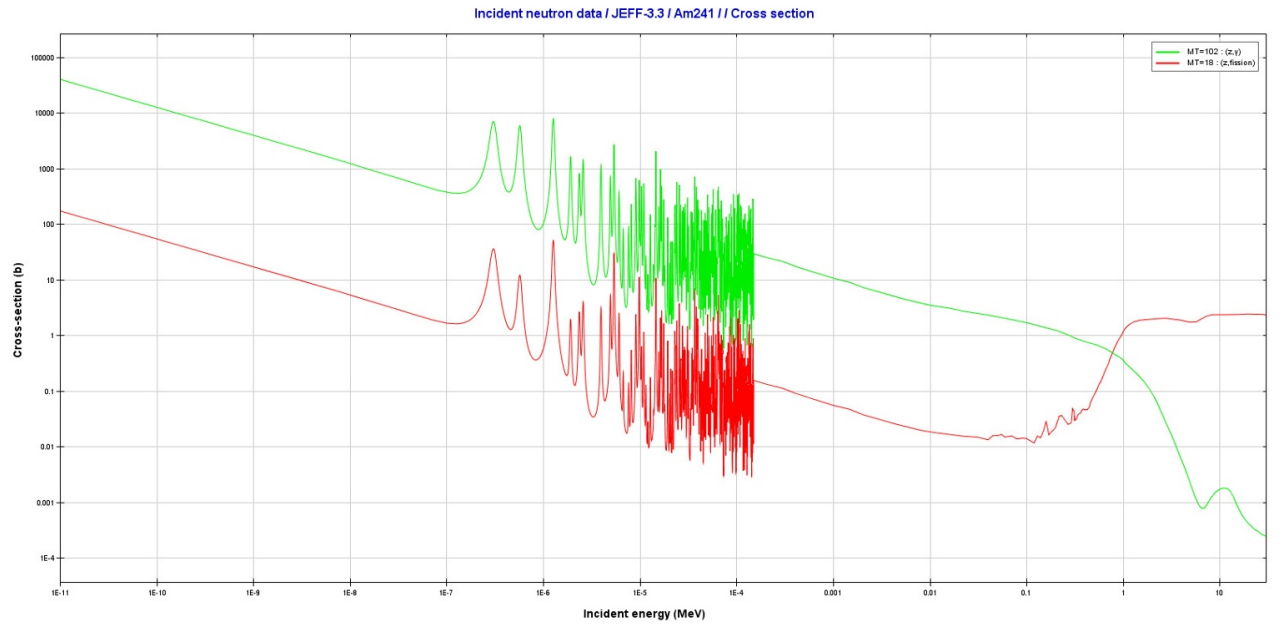

Fig. 2. - Capture and fission cross-section of ${ }^{241} \mathrm{Am}$ as a function of energy.

reactor core $\left\langle\beta_{e f f}\right\rangle$ and due to the fact that minor actinides are having smaller values of delayed neutrons (see table I) compared to uranium isotopes.

The doubling time of the neutron population in the reactor or the period of the reactor being proportional to this delayed neutron fraction:

$$
T=\frac{1}{\rho}\left[\frac{\ell}{K_{\text {eff }}}+\sum_{i=1}^{M} \frac{\beta_{i}}{\lambda_{i}}\right] .
$$

It is obvious that the minor actinides will have a negative effect on the time constants of the system. The higher the amount of minor actinides in the core inventory is, the faster kinetically the reactor becomes. Therefore, with the knowledge and technology of today, we consider only a loading of $2 \%$ to maximum $5 \%$ of minor actinides inventory in a critical sodium fast reactor core.

This would mean that, in order to transmute the legacy waste of more than 40 years of PWR/BWR operation, numerous fast reactors have to be deployed. In a country that wants to continue with nuclear energy, this transition to a fast reactor fleet is credible (since these reactors have other advantages like less uranium resource use). However, in countries that have decided to phase-out nuclear power, the option of building a series of fast reactors for managing their used fuel hence HLW is not an acceptable option.

For those countries in phase out, accelerator-driven systems provide a more attractive solution. Since in these systems the time constants are driven by the accelerator and not by a self-sustained chain reaction, they can be loaded with much more minor actinides: up to $40 \%$ of the core inventory. The Arcas study (Van den Eynde et al. (2013)) [4] has compared the transmutation performances of a sodium fast reactor (SFR) based on presently European SFR design considered in the frame of Gen IV 
TABLE I. - Values of delayed neutron fraction $\beta$ (pcm) for various actinides isotopes.

\begin{tabular}{|c|c|}
\hline Isotope & $\beta(\mathrm{pcm})$ \\
\hline${ }^{235} \mathrm{U}$ & 650 \\
\hline${ }^{238} \mathrm{U}$ & 1480 \\
\hline${ }^{238} \mathrm{Pu}$ & 120 \\
\hline${ }^{239} \mathrm{Pu}$ & 210 \\
\hline${ }^{240} \mathrm{Pu}$ & 270 \\
\hline${ }^{241} \mathrm{Pu}$ & 490 \\
\hline${ }^{242} \mathrm{Pu}$ & 573 \\
\hline${ }^{237} \mathrm{~Np}$ & 334 \\
\hline${ }^{241} \mathrm{Am}$ & 113 \\
\hline${ }^{243} \mathrm{Am}$ & 208 \\
\hline${ }^{242} \mathrm{Cm}$ & 33 \\
\hline${ }^{244} \mathrm{Cm}$ & 100 \\
\hline
\end{tabular}

initiative (3600 MWth/1440 MWe European Fast Reactor) and The accelerator-driven system is based on the lead-cooled 400 MWth European Facility for Indus-trial Transmutation (EFIT) and came with the present results, due to core safety parameters limit the amount of MA that can be loaded in the critical core for transmutation, leading to transmutation rates of

- $\mathrm{SFR}=2$ to $4 \mathrm{~kg} / \mathrm{TWh}$ (depending if one uses heterogeneous or homogeneous MA distribution in the core).

- $\mathrm{ADS}=35 \mathrm{~kg} / \mathrm{TWh}$.

The same FP7 project ARCAS, has shown that for all European countries in phase out, seven $400 \mathrm{MWth}$ ADS units would be required to transmute all minor actinides in a time frame of about 100 years.

If a joint effort is conducted between countries (A) phasing out nuclear energy in EU and countries (B) willing to continue with nuclear energy, with a total of 15 EFIT units, countries A will get their MA completely treated by 2072 if ADS transmutation at industrial scale would start in 2040. In the same time as the same facilities will be shared by countries B, the latest will stabilize their inventory of MA at about 100 tons circulating in the fuel cycle facilities as illustrated in fig. 3 .

The motivation for considering the industrial deployment of partitioning and transmutation $(\mathrm{P} \& \mathrm{~T})$ is manifold; the recycling of the major part of the resources contained in the spent fuel namely uranium and plutonium hence closing the fuel cycle, a better treatment of the residual HLW through advanced separation and transmutation of minor actinides. The latest objective has three main goals: 

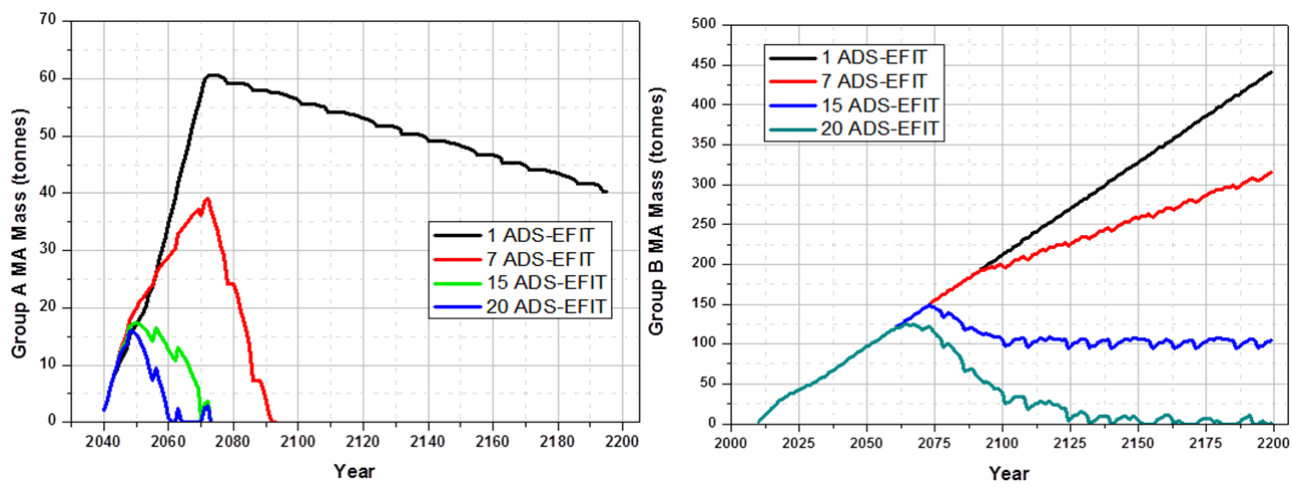

Fig. 3. - Time needed for MA treatment as a function of the number of EFIT units deployed from 2040 on.

- a reduction of the radiological hazard associated with spent fuel by reducing the inventory of minor actinides;

- a reduction of the time interval required to reach the radiotoxicity of natural uranium;

- a reduction of the heat load of the HLW packages to be stored in the geological disposal leading to its efficient use.

P\&T will not replace geological disposal but it is a mean to ease the engineering of such a disposal and to reduce the time frames of this disposal to times that are maybe apprehensible by humans.

The time scale needed for the radiotoxicity of the waste to drop to the level of natural uranium will be reduced (fig. 4) from a "geological" value (500000 to 1 million years) to a value that is comparable to that of human activities (several hundreds of years) (OECD/NEA, 2002, 2006) [5,6].

In 2005, various European actors from R\&D institutions and industries working for more than 30 year in the field of closing the fuel cycle and P\&T came with a global strategy aiming at the industrial deployment by 2035-2040. "The implementation of PEST of a large part of the high-level nuclear waste in Europe needs the demonstration of its feasibility at an "engineering" level. The respective RESD activities could be arranged in four "building blocks":

1) Advanced partitioning of PWR/BWR fuel. This is known technology, much of it already at semi-industrial scale.

2) Fabrication of minor actinide loaded fuel.

3) Transmutation in dedicated industrial transmutation facilities

4) Reprocessing of the transmuted fuel. 


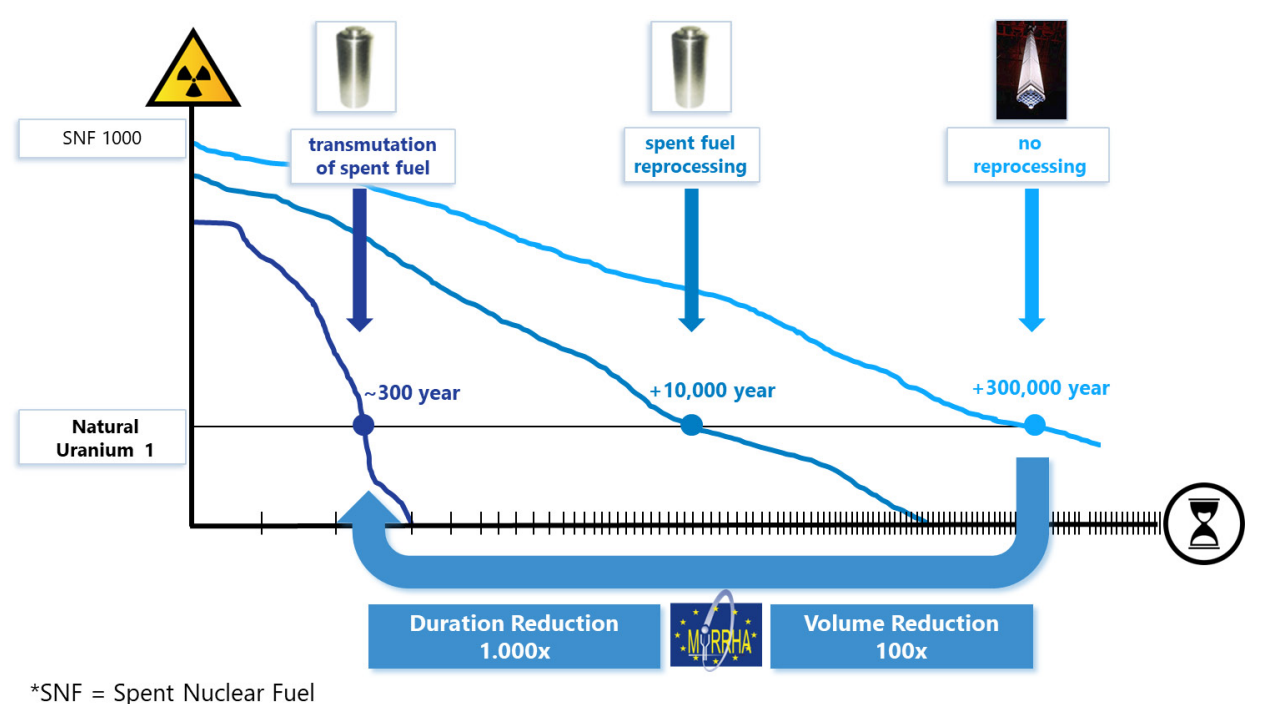

Fig. 4. - Comparison of radiotoxicity of the spent nuclear fuel as a function of time for three situations: a) direct disposal without reprocessing, b) classical PUREX reprocessing and disposal of vitrified HLW including FPs and MAs, c) advanced reprocessing extracting MAs and transmuting them and ultimate disposal only FPs.

MYRRHA aims to be a prototype for the conceptual industrial transmuter, EFIT, designed in the IP-EUROTRANS project (Mansani et al. (2012)) [7].

\section{3. - ADS technology}

The concept of ADS combines a particle accelerator, a spallation target and a nuclear reactor with a subcritical core (see fig. 1). In most proposals, a LINAC proton accelerator is used. The protons are injected by the accelerator onto a spallation target be it a separated unit from the sub-critical reactor or just the heavy liquid metal primary coolant of the reactor, where they produce spallation primary neutrons for driving the subcritical core. Except for the subcritical state, the core of an ADS is very similar to that of a critical reactor. The different components of an ADS are discussed in detail in the following sections.

31. Accelerator. - The accelerator is the driver of the ADS. The accelerator provides the high-energy protons that are used in the spallation target to create neutrons, which feed in their turn the subcritical core. The accelerator can both be a linear accelerator (LINAC) or a circular accelerator (cyclotron). The accelerators used in an ADS must be high-power accelerators and must have an extremely high reliability and availability. These accelerators are under continuous development and the construction of these machines seems feasible. 
Particle accelerators delivering a beam on a target can be organized according to the time structure of their beam:

- Direct Current (DC) beam delivery: this beam with no time structure is typically obtained from a DC accelerator;

- Continuous Wave (CW) beam delivery: in order to be accelerated by radiofrequency (RF) fields, the beam has to be subdivided in small packets called bunches and the $\mathrm{RF}$ has to be synchronized with the bunch progression through the accelerator. If the resulting bunch train is delivered continuously to the target, the beam delivery is called continuous wave. For providing such a beam, the accelerator must operate in a steady state characterized by constant guiding fields and constant RF frequency;

- Pulsed beam delivery: if the bunch train is interrupted in a periodic way (but with a frequency that is much smaller than the RF frequency) a beam on/off cycle is obtained, leading to pulsed operation. Within the pulse, the bunch structure of the beam remains present.

The choice between a LINAC and a cyclotron depends on many factors. In both cases, it is important to specify the beam characteristics for ADS applications, and more specifically the little amount of beam trips accepted. For most ADS the accelerator delivers the particles in continuous mode, although pulsed operation could be used as well (e.g., for testing purposes).

Linear accelerators have the possibility to place the transverse focusing elements (quadrupoles) at frequent intervals along a LINAC, as well as longitudinal focusing, which set a higher limit to the charge per bunch that can be accelerated without significant beam losses. This results in the fact that a LINAC could accelerate a current one to two orders of magnitude higher than a cyclotron, without having problems of beam extraction. The major drawback of a LINAC is its length, which depends on the final beam energy and accelerating gradient. This length of the LINAC is an important factor in the cost of the facility, while most of the accelerator needs to be shielded against radiations.

The base of a cyclotron is the so-called "cyclotron resonance": in a magnetic field, constant in time and perpendicular to the beam orbit, the particle revolution frequency is fixed and independent of the particle energy. The major drawbacks with cyclotrons are the absence of good focusing and the beam extraction. The deflecting system, which guides the beam out of the accelerator, is only permitted to touch only a negligible fraction of the beam. The current limit of the cyclotron is given by a design requirement to produce a clean beam at the outer radius of the machine. The maximum amount of proton energy that can be reached by cyclotrons is limited to less than $1 \mathrm{GeV}$. On a top of that the presently requested reliability in terms of beam trips seems to be out of reach by cyclotrons.

Indeed, the accelerator must have a very high reliability and efficiency. The number of beam trips longer than few seconds should be limited because these beam trips can cause temperature and mechanical stress transients comparable to fast safety rod insertions (SCRAM) in critical reactors. 
3.2. Spallation target. - The spallation target is a neutron source providing primary neutrons that are multiplied by the surrounding subcritical core. The primary neutrons are produced by the spallation reaction of heavy-metal target nuclei, bombarded by the high-energy particles generated by the accelerator.

The spallation target is one of the most stressed components of an ADS. The spallation target is designed to generate the maximum amount of neutrons while it must be able to evacuate the heat generated by the spallation process. In addition, the mixed protonneutron irradiation field that typically can be found in the spallation target of an ADS imposes very specific conditions in the design and operability of the target and influences all thermo-mechanical options for these targets. The target of an ADS must be cooled, mostly by gas, heavy water or liquid metal.

There are two options for the spallation target: a solid target or a liquid metal one. Within the operational, lower-power facilities there is a lot of experience with solid targets, especially when combined with an accelerator which delivers a pulsed beam. These solid targets are difficult to cool and suffer from radiation damage. The target should be cooled further, even if the accelerator is shut off. The path for solid targets for use in an ADS is abandoned in favor for the liquid metal targets.

For higher-power facilities, such as an ADS, the use of a liquid metal spallation target is preferred. Both lead as lead-bismuth eutectic (LBE) are two primary candidates liquid metal target for the production of spallation targets in an ADS. The cooling of a liquid metal spallation target is inherent to the design of the system; there is a constant flow of liquid metal coolant. At the same time, it is possible to achieve higher power densities without having significant irradiation damage to the target. The drawback of a liquid metal target is that the coolant, and especially lead or LBE, can cause erosion or corrosion to the structural materials that are in contact with the coolant.

The use of a liquid metal spallation target introduces some design difficulties. First, we have the choice of materials for the target. The materials must withstand the thermomechanical loads on the targets and must be compatible with the liquid metal. Secondly, an interface between the target and the beam guide must be foreseen. This may take the form of a solid beam window or a windowless design. A beam window is exposed to a significant flux of high-energy protons and neutrons at high temperatures and to a very corrosive environment. Therefore, it is very likely that the beam window must be replaced periodically because of expected material damage. The use of a windowless target where the window is integrated in the beam guide tube will result in approximately 1.5 to 2 times lower radiation dose to this window since neutron irradiation will be negligible in comparison with proton irradiation.

3.3. Subcritical core. - The subcritical core of an ADS is constructed in such a way that it does not have enough fissile material to reach criticality. Source neutrons are needed as an input to drive the system. They are obtained from an energetic beam delivered by the accelerator impinging on a target, through spallation reactions. The subcritical core of an ADS acts as an amplifier: on average $\sim 15$ neutrons are produced for each proton. 
The energy spectrum of the subcritical core depends on the application of the core. For the transmutation of minor actinides, a fast neutron spectrum has various advantages among them a larger excess of neutrons, a reduced amount of minor actinide production in the core and a better energetic yield for eventual energy production.

The evolution of power with time and temperature feedback effects will have an effect on the multiplication factor of the core, as in critical systems. However, for a subcritical core, the behavior is dominated by the external source and its variation in time. The closer to a critical system, the feedback elements of the core will become more important. There are no final criteria to define an optimal level of sub criticality.

Regarding critical cores, reactivity of the core and the power level of the reactor is done through control rods. With an ADS, it should be possible to control the power level of the subcritical reactor through the external source. When a core is working in 'source dominated' mode, the shutdown of the source has an instantaneous effect to reduce power. The inverse effect, a sudden increase of the source has the consequence of an instantaneous increase in the reactor power.

3.4. Advantages of an ADS. - The main advantages of an accelerator-driven system compared to critical reactors are twofold: an ADS allows a greater flexibility with respect to the fuel composition and it has a potentially enhanced safety.

ADS are able to burn fuels that are problematic from the standpoint of critical reactor operation, namely fuels that would degrade neutronic safety characteristics of the critical core to unacceptable levels due to small delayed neutron fractions and short neutron lifetimes. An ADS is capable to cope amongst others with ${ }^{233} \mathrm{U}$ and minor actinide fuel. Additionally, an ADS allows the use of non-fissile fuel such as thorium without the incorporation of $\mathrm{U}$ or $\mathrm{Pu}$ into the fresh fuel.

The enhanced safety of an accelerator-driven system is due to the fact that once the accelerator is turned off, the system shuts down. When the margin towards criticality is sufficiently large, reactivity-induced transients will never result in a super-critical accident.

Since the accelerator is the driver of the system, the power of the ADS is controlled by the beam current of the accelerator. This feature can be utilized to compensate for fuel burnup.

In the context of transmutation, another advantage of the ADS is its non-critical core. This results in increased core design and fuel management flexibility. The sub criticality also opens opportunities for new reactor concepts, including concepts that are otherwise ruled out by insufficient neutron economy. An ADS allows transmuters to be designed as pure transuranic of minor actinide burners. This minimizes the fraction of specialized transmuters in the reactor park.

3.5. ADS developments in the world. - Many ADS concepts have been developed in the world, involving smaller, medium-size or even large installations. The Energy Amplifier developed at CERN in the 1990s was devoted to a better use of natural resources and has evolved then to the XADS (eXperimental ADS) developed by Ansaldo Nucleare. 
At the same time the ADONIS project in Belgium (1995-1996) was solely devoted to the production of radioisotopes for the medical industry; ADONIS evolved to MYRRHA (Multi-purpose hYbrid Research Reactor for High-tech Applications) in 1997; this project still exists and will be detailed in section 5 below.

Both XADS and MYRRHA were regrouped in the frame of the EUROTRANS project of the sixth framework programme from the European Commission (2005-2010). EUROTRANS considered two different but complementary concepts:

- MYRRHA, as smaller scale ADS project for the short term (see below);

- EFIT (for European Facility for Industrial Transmutation), as larger (but still midscale) ADS project for the longer term.

EFIT (Mansani et al. (2012)) [7] comprises a large-scale accelerator (800 MeV, $20 \mathrm{~mA})$ coupled to a pure lead target, the subcritical reactor (400 MWth) being also cooled by liquid lead.

In the meantime (De Bruyn (2012)) [8], the Japanese JAEA was investigating an even larger "commercial-size" installation, with an $(1.5 \mathrm{GeV}, 20 \mathrm{~mA})$ accelerator coupled to an LBE target, the $800 \mathrm{MWth}$ reactor being also cooled by liquid LBE.

At present time, those large-scale facilities have been put on-hold and attention has been focused on the construction and the operation of smaller installations. A zero-power installation, GUINEVERE, has been successfully operated at SCK CEN, since 2011 in the framework of various research projects of the European Commission.

\section{4. - MYRRHA}

4*1. Introduction. - MYRRHA (Aït Abderrahim et al. (2010)) [9], the flexible experimental accelerator-driven system in development at SCK CEN, will demonstrate the ADS full concept by coupling the three components (accelerator, spallation target and subcritical reactor) at reasonable power level to allow operation feedback, scalable to an industrial demonstrator and allow the study of efficient transmutation of high-level nuclear waste. The MYRRHA-facility is conceived as a flexible irradiation facility, able to work in both subcritical and critical modes. Both modes of operation have their specific energy and flux distributions, which permit, besides transmutation experiments, a wide range of applications, from fuel developments for innovative reactor systems, material developments for Gen IV systems, material developments for fusion reactors, to radioisotope production for medical and industrial applications. Since MYRRHA is based on the heavy liquid metal technology, lead-bismuth eutectic, it will be able to significantly contribute to the development of Lead Fast Reactor (LFR) Technology and in critical mode, MYRRHA will play the role of European Technology Pilot Plant in the roadmap for LFR.

4. The MYRRHA accelerator. - The accelerator is the driver of MYRRHA while it provides the high-energy protons that are used in the spallation target to create neutrons that in turn feed the subcritical core. The accelerator must be able to provide a proton 


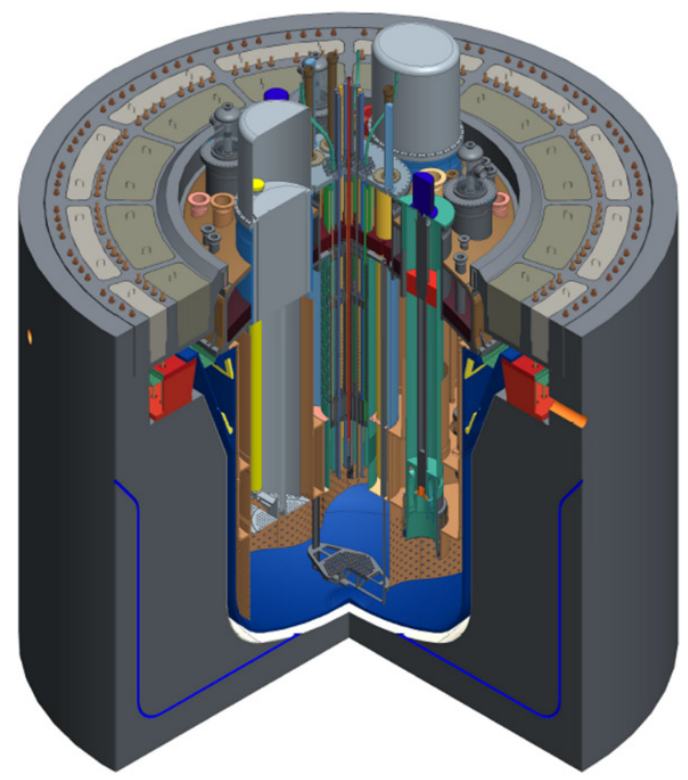

Fig. 5. - 3-D Schematic view of the MYRRHA sub-critical reactor.

beam with energy of $600 \mathrm{MeV}$ and a maximum current of $4 \mathrm{~mA}$, which will be delivered to the core in continuous wave mode. The beam is delivered to the core from above through a beam window.

Accelerator availability is a crucial issue for the operation of the ADS. A high availability is expressed by a long Mean Time Between Failure (MTBF), which is commonly obtained by a combination of over-design and redundancy. On top of these two strategies, fault tolerance must be implemented to obtain the required MTBF. Fault tolerance will allow the accelerator to recover the beam within a beam trip duration tolerance after failure of a single component. In the MYRRHA case, the beam trip duration tolerance is 3 seconds. Within an operational period of MYRRHA the number of allow beam trips exceeding 3 seconds must remain under 10, shorter beam trips are allowed without limitations (Rimpault et al. (2012)) [10]. The combination of redundancy and fault tolerance should allow obtaining a MTBF value in excess of 250 hours.

At present proton accelerators with megawatt level beam power in $\mathrm{CW}$ mode only exist in two basic concepts: sector-focused cyclotrons and linear accelerators. Cyclotrons are an attractive option with respect to construction costs, but they do not have any modularity which means that a fault tolerance scheme cannot be implemented. Also, an upgrade of its beam energy is not a realistic option. A linear accelerator, especially if made superconducting, has the potential for implementing a fault tolerance scheme and offers a high modularity, resulting in the possibility to recover the beam within a short time and increasing the beam energy. 


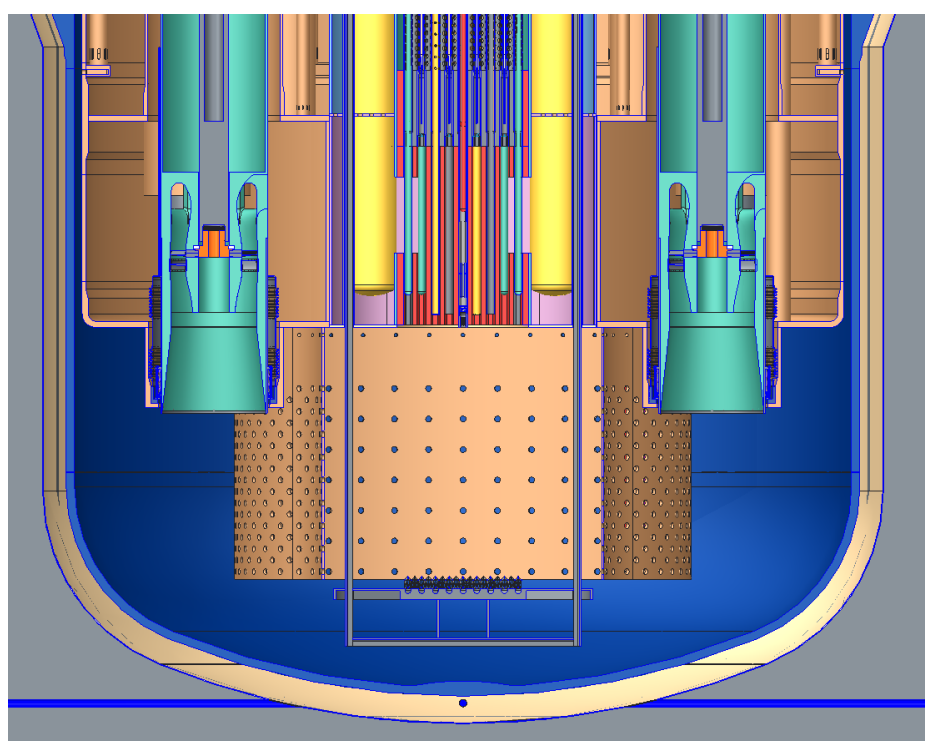

Fig. 6. - MYRRHA reactor core with reactor experiments and spallation target in the red zone.

4.3. The MYRRHA reactor. - MYRRHA consists of a pool-type ADS with the ability to operate in critical mode cooled by liquid lead-bismuth eutectic. Consequently, all the primary systems are housed within the reactor vessel. The reactor is located in the reactor pit (fig. 5) which features a liner able to serve as secondary containment in case of a reactor vessel leakage or break. The reactor cover closes the reactor vessel and supports all the components.

The reactor core (fig. 6) is included in the core barrel and consists of 211 positions. Such positions are filled, among others, with fuel assemblies, control rods, scram rods, the spallation window, in-pile sections, reflector assemblies, instrumentation and surveillance capsules. From these core positions 55 are accessible from the cover and are called Multifunctional Channels (MFC).

In the multifunctional channels (MFC) control rods, scram rods, spallation window, in-pile sections, instrumentation and surveillance capsules are installed from the top. Because of the structures above the core, the fuel and reflector assemblies have to be loaded from underneath. Thanks to the buoyancy, the fuel and reflector assemblies do not need locking devices to hold them on the core support structure. However, in order to avoid any small position changes of the fuel assemblies inside the core during operation, a core restraint system fixes the radial position of the fuel assemblies.

Two in-vessel fuel-handling machines, installed permanently in the reactor, handle the loading and the unloading of the fuel assemblies to the in-vessel fuel storages, which are integrated into the diaphragm. Due to the chosen manipulator concept, the most compact design is obtained by using two independent machines, each covering the half of the core and serving the half of the in-vessel fuel storage positions. The diaphragm 
separates the cold, high-pressure LBE from the hot, low-pressure LBE and contains the in-vessel fuel storages and the pump casings.

The diaphragm is connected, together with the cover, to the reactor vessel. The diaphragm consists of two horizontal plates connected to each other by vertical shells allowing some components to reach the lower plenum. Due to this design, several volumes exist between the two horizontal plates. Two of these volumes house part of the pumps and the heat exchangers. Each pump casing lodges one pump and two heat exchangers. Four volumes are dedicated for the in-vessel fuel storage. These storages are cooled during operation mainly by the forced circulation of LBE imposed by the pumps, while during shutdown cooling is ensured by natural circulation.

The four primary heat exchangers extract the power of the primary system exchanging it with the secondary system. The head imposed by the pumps aspirates the hot LBE from the hot plenum into the heat exchangers. After being cooled, the LBE flows into the pump casings and then through the pumps themselves. This concept mitigates the LBE corrosion by reducing the temperature of the fluid inside of the pumps. In particular, these are sized in order to be able to pump the LBE through the core balancing also the head losses of all the other sections of the primary system. The number of heat exchangers and pumps is optimized in function of the space utilization inside the reactor vessel and the symmetry of the coolant flow. This leads to a configuration with two heat exchangers and one pump on each of the two sides of the reactor.

4.4. RED in support to design and licensing. - SCK CEN conducts since the beginning of the programme an ambitious $R \& D$ programme to address the main design and licensing challenges. In this frame SCK CEN has constructed and commissioned various LBE test facilities which are used for qualification of the key materials and components of MYRRHA. These facilities can also be used for the development of materials and components for fast reactors of all power ranges, including SMR-type systems, working with LBE or lead as coolant.

The main challenges of the MYRRHA development are in particular related to the use of liquid Lead-Bismuth Eutectic as reactor coolant. Consequently various LBE test facilities for research on

- heavy liquid metal chemistry and conditioning,

- heavy liquid metal corrosion of materials,

- thermal hydraulics in heavy liquid metals,

- instrumentation in heavy liquid metals, and

- testing of components in heavy liquid metals

have been constructed and commissioned (Fernandez et al. (2019)) [11].

For the components and thermal hydraulics programme the E-SCAPE facility, European SCAle Pool Experiment, a thermal hydraulic 1/6-scale model of the MYRRHA reactor, has been commissioned in spring 2017. The main objective of this installation 


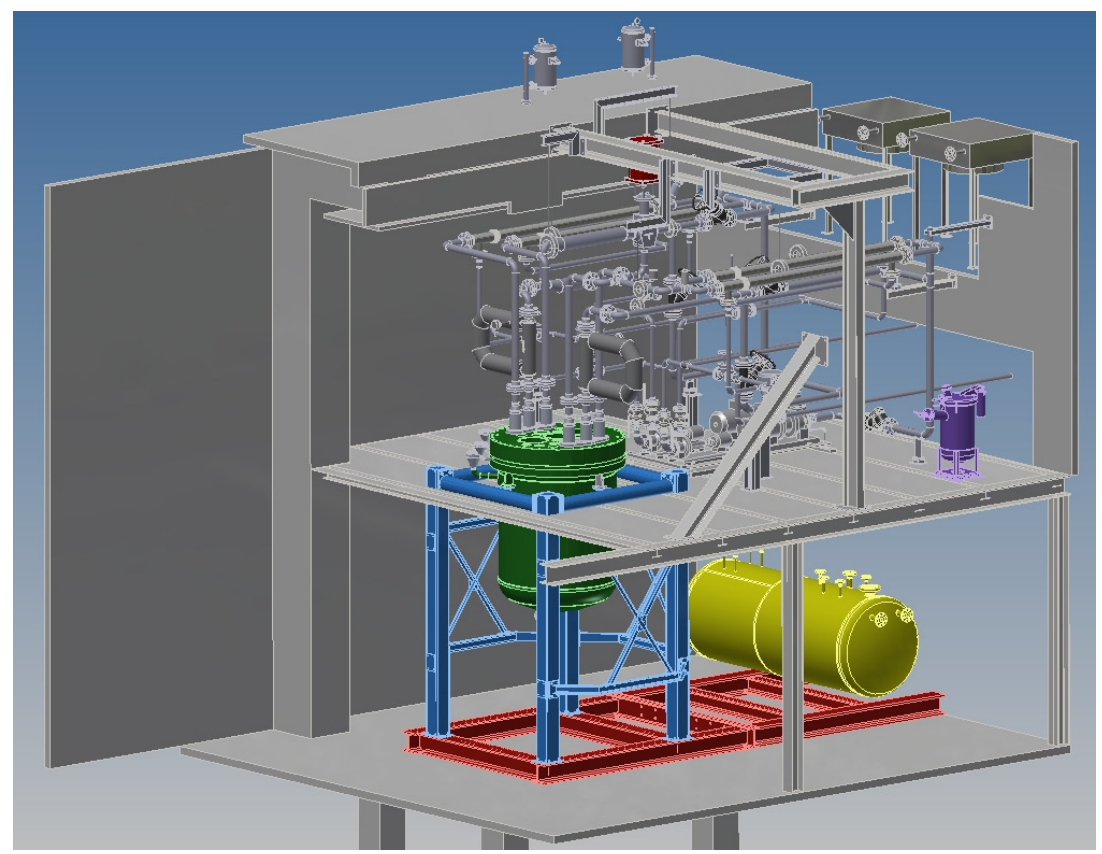

Fig. 7. - E-SCAPE facility representing a 1/6 Mock-up of MYRRHA reactor with all its in-vessel internals and electric heating of $15 \mathrm{~kW}$ for the decay heat simulation of the core.

(fig. 7) is the study of the thermal hydraulic behavior of liquid metal in a complex pool geometry. The characterization of the pool thermal hydraulic phenomena is needed for the code qualification of computational fluid dynamics and other system tools used for the safety assessment of a reactor system.

The COMPLOT, COMPonents LOop Testing, facility is a large-scale, closed-loop isothermal experimental loop in operation since 2014, used for hydraulic experiments of full-scale reactor components in flowing LBE. The goal of COMPLOT is to simulate a full-scale hydraulic flow path through the MYRRHA core allowing the characterization of hydraulic and hydrodynamic behavior of full-scale MYRRHA core components such as the fuel assembly, the spallation target, the control and safety rod systems and other in-pile instrumentation, systems and experiments.

HEXACOM, Heat EXchAnger at COMplot, steam loop is a two-phase water-steam cooling circuit that provides temperatures and flow conditions representative of the MYRRHA Secondary Cooling System. The main objectives of the facility is to investigate the heat transfer performances of different PHX configurations within the operational ranges relevant to MYRRHA, to develop and validate heat transfer correlations specific to bayonet tube applications in flowing LBE, to improve the level of knowledge on the phenomena that occur in the steam/water side of double wall bayonet tube heat exchangers operated at low pressure, to obtain experimental data suitable for model development 
and/or the validation and verification of system codes. Since HEXACOM is a small version of the MYRRHA secondary and tertiary system, additional phenomena, typical for steam/water, natural circulation cooling and anti-freezing strategies can be studied in support to the design of these systems in MYRRHA.

Finally, RHAPTER, the Remote HAndling Proof-of-principle TEst Rig, in operation since 2011, is designed to test and validate mechanical components submerged in LBE.

The LBE chemistry and conditioning programme uses the MEXICO, Mass EXchanger In Continuous Operation, loop to test different oxygen control systems, such as the gas phase, solid oxide phase and electrochemical oxygen pumping systems for regulating dissolved oxygen in liquid lead-bismuth eutectic. It is also used to evaluate the efficiency and expected life time of filtration systems for purifying the LBE of oxides and possible impurities from the liquid metal flow while minimizing the created heavy metal waste stream.

HELIOS, HEavy LIquid metal Oxygen conditioning System, is a LBE conditioning and storage setup which is used to investigate LBE conditioning schemes. It can also serve to study calamity mitigation strategies after a possible steam ingress due to a tube rupture in a heat exchanger or exposure to air.

The main infrastructure for materials research is CRAFT, Corrosion Research for Advanced Fast reactor Technology. It is an installation for long-term corrosion experiments on MYRRHA candidate materials in liquid LBE. The loop type installation operates at representative conditions of temperatures, LBE velocities and dissolved oxygen concentrations of MYRRHA.

LIMETS, LIquid MEtals Test Stands, are experimental setups designed for mechanical testing of materials in a stagnant LBE environment in order to investigate mechanisms and kinetics of material-liquid metal interactions that influence the mechanical properties of materials.

\section{5. - Conclusions}

In order to reduce the amount of long-lived radioactive waste legacy and for the countries willing to continue the use of nuclear energy in their energy mix with limited amount of natural resources, the fuel cycle of the nuclear energy systems needs to be closed for a better use of the uranium and recycling the plutonium.

Partitioning and transmutation is an attractive solution to reach the goal of sustainable nuclear energy systems. P\&T could address the high-level radioactive waste issue by going towards advanced separation of minor actinides and their transmutation by fission. This will initiates the strategy towards a more resource-efficient nuclear energy system in the future. P\&T will not replace the need for appropriate geological disposal of high-level waste, but it will reduce the long-term radiotoxicity of the waste significantly and thus reduce the burden of HLW on the safety of geological disposal increasing highly its acceptability by the public.

All transmutation strategies with closed fuel cycles could achieve high reductions in the inventory of actinides and the radiotoxicity of the nuclear waste by making use of fast 
neutron spectrum systems. The latest being fast critical reactor or accelerator-driven systems.

Nevertheless, the sub-critical ADS offers interesting degrees of freedom with respect to the core design and fuel management as well as flexibility to moving policies towards the use of nuclear energy.

With the extensive development of accelerator technology, it appears today that proton beam powers of $10 \mathrm{MW}$ to $100 \mathrm{MW}$ with the needed reliability for operating ADS systems are within the reach in the coming decade with the LINAC technology.

\section{REFERENCES}

[1] DOE/EIA, International Energy Outlook 2019 (U.S. Energy Information Administration, Washington, DC) 2019, website: https://www.eia.gov/outlooks/ieo/.

[2] OECD, OECD Factbook 2015-2016: Economic, Environmental and Social Statistics (OECD Publishing) 2016, website: http://www .oecd.org/publications/oecd-factbook$18147364 . \mathrm{htm}$.

[3] Uranium 2018: Resources, Production and Demand. A joint report by the OECD Nuclear Energy Agency and the International Atomic Energy Agency, NEA\#7413 (OECD/IAEA) 2018, available online at http://www.oecd-nea.org/ndd/pubs/2018/7413-uranium2018.pdf.

[4] ARCAS: ADS and fast Reactor CompArison Study, project reference: 249704, funded under: FP7-EURATOM-FISSION.

[5] Accelerator-Driven Systems (ADS) and Fast Reactors (FR) in Advanced Nuclear Fuel Cycles, A Comparative Study (OECD/NEA) 2002, ISBN 92-64-18482-1.

[6] Physics and Safety of Transmutation Systems, A Status Report (OECD/NEA) 2006, ISBN 92-64-01082-3.

[7] Mansani L., Artioli C., Schikorr M. et al., "The European Lead-Cooled EFIT Plant: An Industrial-Scale Accelerator-Driven System For Minor Actinide Transmutation", Nucl. Technol., 180 (2012) 241.

[8] DE BRUYN D., "Different ADS concepts in the world: EFIT, MYRRHA \& the others", LEADER Technical Workshop Design and safety analysis of ALFRED, Pisa (Italy), September 2012 (CIRTEN (Interuniversity Consortium for Technological Nuclear Research)) 2012.

[9] Aït Abderrahim H., Baeten P., De Bruyn D. et al., "MYRRHA, a Multipurpose hYbrid Research Reactor for High-end Applications", Nucl. Phys., 20 (2010) 24.

[10] Rimpault G., Darde P., Mellier F. et al., "The issue of accelerator beam trips for efficient ADS operation", Nucl. Technol., 184 (2013) 249.

[11] Fernandez R., Van Tichelen K., Aerts A. et al., "MYRRHA Technology \& Research facilities in support of Heavy Liquid Metal SMR fast Reactors", IAEA Technical Meeting on Fast Reactors SMR, Milan (Italy), 25-27 September 2019, to be published as IAEA TECDOC, 2019. 